\title{
Desarrollo de un sistema lógico difuso para el control de la locomoción bípeda de un robot humanoide NAO
}

\author{
Karen Lizbeth Flores-Rodríguez, Felipe Trujillo-Romero \\ Universidad Tecnológica de la Mixteca, \\ División de Estudios de Posgrado, Huajuapan de León, Oaxaca, \\ México \\ karenflores350@hotmail.com,ftrujillo@mixteco.utm.mx
}

\begin{abstract}
Resumen. En el siguiente trabajo se desarrolla un sistema lógico difuso para el control de la locomoción bípeda de un robot humanoide NAO. Se considerando como entradas las posiciones de los ángulos de las piernas y como salida la posición cercana a cero del ángulo del torso ambas con respecto a un marco de referencia local independiente. El trabajo parte del modelo Walking Toy el cual es un sistema dinámico que describe la locomoción de un bípedo. Se planea evadir la complejidad del sistema dinámico y se propone un sistema difuso de inferencias que permita estabilizar una trayectoria de caminado para el sistema. Los resultados son aceptables, se obtuvo la simulación del caminado estable para el robot NAO.
\end{abstract}

Palabras clave: Lógica difusa, locomoción bípeda, Walking Toy, robot NAO.

\section{Introducción}

La investigación sobre locomoción de robots bípedos es el estudio y desarrollo de métodos para lograr estabilidad y equilibrio en el caminado basado en el caminado humano. Este caminado se analiza como una órbita periódica de una fase estable que alterna con una fase inestable. El caminado, por definición, es moverse a un paso moderado levantando y poniendo un pie delante de otro en turnos mientras el otro pie está sobre el suelo. Al menos un pie debe estar tocando el suelo en todo momento para considerarse caminado. El objetivo del análisis de locomoción de robots bípedos es diseñar movimientos de articulaciones y controladores asegurando que este no caiga al caminar. El caminado bípedo se realiza como un conjunto de posiciones de ángulos deseados en el tiempo para las articulaciones de las piernas, conocido también como patrón de caminado. El caminado se considera estable si el único contacto entre el bípedo y el piso es realizado con las plantas de los pies.

El marco básico del control de la locomoción bípeda es el generador de patrones de caminado y la estabilización de los mismos. En este trabajo se 
propone un control lógico difuso para la generación de dichos patrones y la estabilizacion de la locomoción bípeda. Se planea evadir la complejidad de los sistemas dinámicos y se propone un sistema difuso de inferencias que permita estabilizar una trayectoria de caminado. Utilizando lógica difusa se proporciona un mecanismo de inferencia que permite simular los procedimientos de razonamiento humano en sistemas basados en el conocimiento. El trabajo parte de las restricciones que se plantean en la aplicación del modelo Walking Toy [16], para la locomoción de robots bípedos. Este modelo se describe en una sección posterior del trabajo.

La teoría de la lógica difusa proporciona un marco matemático que permite modelar la incertidumbre de los procesos cognitivos humanos de forma que pueda ser tratable por una computadora. Básicamente la lógica difusa es una lógica multivaluada que permite representar matemáticamente la incertidumbre y la vaguedad, proporcionando herramientas formales para su tratamiento. Se dice que, cualquier problema del mundo puede resolverse como: dado un conjunto de variables de entrada (espacio de entrada), se debe obtener un valor adecuado de variables de salida (espacio de salida). La lógica difusa permite establecer este mapeo de una forma adecuada, atendiendo a criterios de significado (y no de precisión). El documento se organiza como sigue. En la sección 2 se presenta el estado del arte referente principalmente a contribuciones con el robot humanoide NAO. En la sección 3 se describe brevemente el Control Óptimo Difuso para la resolución de sistemas dinámicos. en la sección 4 se presenta el Sistema de Locomoción de Robots Bípedos donde se explica el modelo Walking Toy. En la sección 5 se presenta el Desarrollo del Controlador Lógico Difuso que incluye la Fuzzificación, Inferencia y Defuzzificación. En la sección 6 se plasman las pruebas y resultados del trabajo. Se muestra el diseño del controlador difuso con el toolbox de MATLAB, la herramienta Simulink y una simulación en Webots. Finalmente, en la sección 7 se dan algunas conclusiones del presente trabajo.

\section{Estado del arte}

Desde que la plataforma NAO [1] fue elegida como plataforma estándar de la competencia de fútbol robótico RoboCup [11], las investigaciones en el área de locomoción bípeda por parte de universidades y centros de investigación en todo el mundo se incrementaron, pues este robot permite a los investigadores enfocarse en la creación de algoritmos de resolución para los problemas típicos de los robots humanoides y dejar un poco de lado el desarrollo de plataformas robóticas. Entre lo resaltable de la liga de plataforma estándar de RoboCup se encuentran los siguientes trabajos. En [7], se presentan una sólida marcha de circuito cerrado. Basado en el análisis y control del centro de masa soportada por métodos de balanceo. En [4], utilizan el método del péndulo invertido para la generación de movimientos del robot y el criterio del punto de momento cero ZMP para asegurar la buena ejecución de los movimientos. Con una velocidad de $25 \mathrm{~cm} / \mathrm{seg}$, es uno de los más rápidos. En [8], presentan una marcha que radica en el uso del modelo del péndulo invertido con duración de fase dinámica para el 
Desarrollo de un sistema lógico difuso para el control de la locomoción bípeda de un robot ...

modelado de un rápido y robusto caminado omnidireccional, responsivo y reactivo a perturbaciones externas. Con una velocidad de $31 \mathrm{~cm} / \mathrm{seg}$ hacia adelante y un paso de $7 \mathrm{~cm}, 12 \mathrm{~cm} / \mathrm{seg}$ de lado con un paso de $8 \mathrm{~cm}, 22 \mathrm{~cm} / \mathrm{seg}$ hacia atrás y a $92^{\circ} / \mathrm{seg}$ cuando rota en su lugar. En [9], se describe una locomoción omnidireccional, con una velocidad hacia adelante de $34 \mathrm{~cm} / \mathrm{seg}$. Este caminado implementa la dinámica del péndulo invertido y el criterio de punto de momento cero y el enfoque de descomposición de la marcha en plano dinámico sagital y coronal, para después recombinarlos sincronizadamente.

Otro trabajos relacionados con el robot NAO, como [5], desarrolla un algoritmo sobre locomoción bípeda llamado Sammy's Walks. Se basa en estudios de frecuencia, aprovecha las oscilaciones propias del sistema para determinar los ciclos límite y generar la locomoción mas eficiente. Este inspirado en los caminantes pasivos, no entrega el caminado esteticamente estable pero si un gran ahorro energía. Por otro lado, en [14], se presenta una técnica que encuentra automáticamente modos de caminar estables y rápidos. Modelizando la caminata como ondas acopladas reduce el espacio de parámetros y mantiene gran diversidad de movimientos. Otra investigación presentada en [3], describe un enfoque libre de modelo donde utilizan un controlador compuesto por neuronas lineales y no lineales. Se obtiene retroalimentación desde las señales de salida para generar mejores señales y mejorar la estabilidad del caminado. El movimiento de los brazos es utilizado para hacer el caminado suave y robusto. En [10], se introduce el modelo predictivo, el cual es capaz de manejar las restricciones dinámicas que puede presentar el punto de momento cero. Este enfoque es adaptable a cualquier plataforma robótica, como el robot HRP-2 y el robot NAO. En [13], se describe un ciclo abierto de caminado en diferentes pendientes. En el cual la planeación de trayectoria es presentada usando las ecuaciones de semi elipse de movimiento. Este enfoque alcanza la velocidad de caminado de $17 \mathrm{~cm} / \mathrm{s}$.

Los métodos de computación flexible como las redes neuronales y la lógica difusa se han vuelto populares en la resolución de dinámicas de sistemas complejas. La lógica difusa ha sido aplicada con éxito para la generación de locomoción para robots. Trabajos como [2], [15], [19], [12], [6] y [17] utilizan lógica difusa para la generación de patrones de caminado y estabilización del mismo. En [18] se describe un controlador omnidireccional de caminado bípedo basado en lógica difusa para el robot NAO. Este controlador se encarga de la restriccion de la longitud de paso e inclinación del cuerpo del robot, tomando como entradas la direccion deseada y ángulo de rotación. El criterio ZMP es utilizado como base para la restricción de la ubicación de la planta del pie del robot.

En resumen, el estudio de la locomoción bípeda puede resolverse mediante diferentes enfoques siendo los más populares el enfoque de péndulo invertido y el criterio ZMP para asegurar la estabilidad. La investigación en esta área radica en las variantes de los enfoques para la resolucíon óptima, mejora de estabilidad y reacción a perturbaciones externas. Se debe considerar que cada robot humanoide implementa el método de resolución para locomoción bípeda que más se adapte a su arquitectura y configuracion. En cuanto a las marchas generadas para el robot NAO, la constante de investigación y desarrollo se enfoca 
en mejorar la estabilidad y aumentar la velocidad para fines de fútbol robótico, los algorimtos deben ser rápidos y no deben consumir demasiados recursos pues se deben solucionar otros problemas como navegación, reconocimiento de objetos, comunicación, entre otros.

\section{Control óptimo difuso}

El control estándar se basa en el conocimiento de ciertas ecuaciones diferenciales $\dot{x}=f(x, u)$ con $x\left(t_{0}\right)=x_{0}$ que describen la dinámica del sistema. Si no se conoce, entonces se puede usar cierto conocimiento para proponer leyes de control. Por ejemplo:

$$
R_{j}: \text { if }(x, u) \text { is } A_{j} \text { then } x \text { is } B_{j}, j=1,2, \ldots, r .
$$

A lo anterior se le conoce como control difuso, el cual es un camino para y transformar conocimiento lingüístico en leyes de control. Para esto se considera un proceso de tres pasos:

1. Modelado de conceptos difusos en reglas.

2. Elección de conectividades difusas en reglas.

3. Elección de procesos de defuzzificación.

Considerando un sistema MISO (múltiples entradas una salida) de reglas donde la variable de entrada es: $x=\left(x_{1}, x_{2}, \ldots, x_{n}\right) \epsilon X=\left(X_{1}, X_{2}, \ldots, X_{n}\right)$ y la variable de salida es $u=Y$. Primero se especifican particiones difusas de todo el espacio involucrado $A_{j}$ y $B_{j}$ en $X$ y $Y$ correspondientes a variables lingüísticas como small positive. Después se definen las reglas IF-THEN correspondientes a esas particiones difusas de expertos o información de entrenamiento. El procedimiento se define en los siguientes tres pasos:

Paso 1: $R_{j}$ : if $x$ is $A_{j}$ then $u$ is $B_{j}, j=1,2, \ldots, r$ Las variables lingüísticas A'js y B'js son subconjuntos difusos de espacios apropiados. En el espacio expertos para $x_{j} \epsilon X, i=1,2, \ldots, m$ se tienen $N$ expertos con la proposición $x_{i}$ is $A$ verdadero. El grado de $x_{i}$ en $A, A\left(x_{i}\right)$ es $M / N$ donde $M$ es el número de expertos. Las reglas de control deben contener modificadores difusos como muy o casi todo, etc. En base a la información de los expertos se definen las funciones de membresía y se especifican los parámetros de las funciones.

Paso 2: Después de modelar las etiquetas lingüísticas en reglas como conjuntos difusas de conjuntos apropiados se seleccionan las conectividades lógicas como t-norma (min o producto), t-conorma (max o doble producto) o negación $(1-\mathrm{x})$.

Paso 3: Teniendo un panorama de salidas difusas $B$ como un subconjunto difuso del espacio de salida, se debe resumir en una sola variable $u *$ a usar como control de acción actual. Es necesario elegir un proceso de defuzzificación $D:[0,1]^{U} \rightarrow U$. En la Tabla 1 se muestran algunos ejemplos de proceso de defuzzificación. 
Desarrollo de un sistema lógico difuso para el control de la locomoción bípeda de un robot ...

Tabla 1. Procesos de defuzzificación.

\begin{tabular}{lc}
\hline Método & Función \\
\hline Centroide & $u *=\frac{\int_{U} u B(u) d u}{B(u) d u}$ \\
Media de la máxima & $u *$ eselpromediodeloselementosdeU \\
Centro de área & $u *$ eselvalormediodelareatotaldeB \\
\hline
\end{tabular}

\section{Sistema de locomoción de robots bípedos}

Para evitar la complejidad de la dinámica de las piernas del robot humanoide NAO se considera un sistema simple. El sistema parte del enfoque Walking Toy[16] el cual se puede apreciar en la Figura 1. Este modelo cuenta con dos piernas de masa $M_{l}$ y longitud idéntica $L$, conectadas a una rueda (cuerpo) inercial de masa $M_{W}$ mediante dos motores rotatorios que provee el torque necesario para mover las piernas. Ambas piernas se encuantran conectadas al eje central del cuerpo rodante, el cual se considera la cadera de la máquina. El modelo matemático para este sistema se presenta en (2).

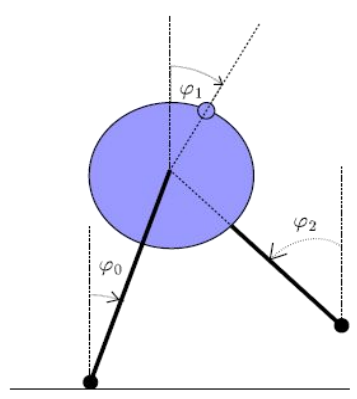

Fig. 1. Walking Toy esquemático.

$$
\begin{gathered}
J_{0} \ddot{\varphi}_{0}=m g L \sin \varphi_{0}-\tau_{1}, \\
J_{1} \ddot{\varphi}_{1}=\tau_{1}-\tau_{2}, \\
J_{2} \ddot{\varphi}_{2}=\tau_{2},
\end{gathered}
$$

donde $J_{0}$ es la inercia de la pierna apoyada y el cuerpo con respecto al punto pivote del suelo. $J_{1}$ y $J_{2}$ representan la rotación inercial del cuerpo y la pierna balanceada con respecto a la cadera. Los torques aplicados, actuando como control son denotados por $\tau_{1}$ y $\tau_{2}$. Sabiendo que $m=M_{l}+M_{W}$. Cuando la pierna balanceada aterriza en el suelo, el modelo del sistema cambia los roles de las piernas. 
Del modelo esquemático del sistema, es evidente la existencia de ciertas restricciones que deben ser consideradas para la realización de un caminado bípedo:

1. El ángulo del pie apoyado sobre el suelo debe ser pequeño y no debe pasar de cierto rango, $\left|\varphi_{0}\right|<\frac{\pi}{2}$,

2. La pierna balanceada nunca tendrá la punta extrema por debajo del nivel del suelo horizontal, siempre será positiva $\left|\varphi_{0}(t)\right| \leq\left|\varphi_{2}(t)\right| \forall t$.

Además de las restricciones antes mencionadas, se conocen ciertas características del sistema. Teniendo en cuenta que el objetivo es que el sistema ejecute un paso suavemente desde una posición de reposo del tiempo $t=t_{0}$ al tiempo $t=T>t_{0}$. Se Considera como posición de reposo aquella en la cual el ángulo de la pierna apoyada contra el suelo $\varphi_{0}$ es simétrica al ángulo de la pierna balanceada $\varphi_{2}(3)$ :

$$
\varphi_{0}=-\varphi_{2}
$$

Teniendo en cuenta que el sistema considera los torques de los eslabones de las piernas como entradas de control, se define la trayectoria en base a los ángulos de las piernas del bípedo. La posición inicial de pie es determinada por el ángulo inicial arbitrario de la pierna fija. Este debe ser un ángulo positivo (4):

$$
\varphi_{0}\left(t_{0}\right)=\alpha .
$$

Al final del primer paso el bípedo debe alcanzar una nueva posición de pie caracterizada por la pierna balanceada (5):

$$
\varphi_{0}(T)=-\beta, \beta>0 .
$$

La posición inicial de los pies al tiempo $t_{0}$ es entonces (6):

$$
\begin{gathered}
\varphi_{0}\left(t_{0}\right)=-\alpha, \\
\varphi_{2}\left(t_{0}\right)=\alpha, \\
\varphi_{1}\left(t_{0}\right)=\frac{J_{0}}{J_{1}}\left(1-\frac{J_{2}}{J_{0}}\right) \alpha .
\end{gathered}
$$

Similarmente al tiempo $t=T>0$, tiempo en completarse el primer paso, el estado del sistema debe satisfacer la siguiente relación (7):

$$
\begin{gathered}
\varphi_{0}(T)=\beta, \\
\varphi_{2}(T)=-\beta, \\
\varphi_{1}(T)=-\frac{J_{0}}{J_{1}}\left(1-\frac{J_{2}}{J_{0}}\right) \beta .
\end{gathered}
$$

Una vez que se complete el primer paso en el intervalo de tiempo $\left[t_{0}, T\right]$ es necesario permutar la pierna apoyada al suelo con la pierna balanceada al tiempo $t=T$. De esta manera bastará con tomar el mismo modelo pero cambiando los valores de $\varphi_{0} *$ y $\varphi_{2} *$. Entonces se puede comenzar en el tiempo $T$ un nuevo paso con el mismo modelo al estado estable con $\varphi_{0}=\beta$ y $\varphi_{2}=-\beta$ (8):

$$
\begin{gathered}
J_{0} \ddot{\varphi}_{0}=m g L \sin \varphi_{0}-\tau_{2}, \\
J_{1} \ddot{\varphi}_{1}=\tau_{2}-\tau_{1}, \\
J_{2} \ddot{\varphi}_{2}=\tau_{1} .
\end{gathered}
$$


Desarrollo de un sistema lógico difuso para el control de la locomoción bípeda de un robot ...

Las condiciones finales de reposo del primer paso se convierten en las condiciones iniciales del segundo paso, las cuales pueden ser de longitud arbitraria. Se considera el intervalo $\left[T=T_{1}, T_{2}\right]$.

\section{Desarrollo del controlador lógico difuso}

\subsection{Fuzzificación}

La Fuzzificación considera dos variables de entrada para el sistema de locomoción bípeda: el ángulo de la pierna apoyada al suelo y el ángulo de la pierna balanceada. Estos ángulos se intercalan conforme se ejecuta el caminado, por comodidad se define ángulo 1 como pierna derecha y ángulo 2 como pierna izquierda. Tomando en cuenta las restricciones mencionadas en la sección de Locomoción de Robots Bípedos, se considera que ambos ángulos de las piernas no pueden tener valores mayores al rango $-45^{\circ}$ a $45^{\circ}$, otra restricción es que los ángulos solo serán iguales en $0^{\circ}$ que es la posición de pie. Con estas restricciones se procede a modelar las funciones de membresía para la variable de salida que es definida como el ángulo de inclinación de un punto en el torso del bípedo que debe ser cercano a cero.

Para cada una de las piernas se consideran cinco funciones de membresía de tipo Gaussiana ya que que el caminado bípedo es un ciclo continuo y suave. Las funciones de membresía se definen entre $-45^{\circ},-20^{\circ}, 0^{\circ}, 20^{\circ}$ y $45^{\circ}$. Estas son las posiciones del pie: atrás, menos atrás, centro, menos adelante y adelante. Las funciones Gaussianas tienen diferentes amplitudes, estas se definen a continuación:

$$
x_{1}, x_{2}=\left\{\begin{array}{lc}
\text { atras } & e^{-\frac{1}{2}\left(\frac{x-(-45)}{12}\right)^{2}} \\
\text { menos }- \text { atras } & e^{-\frac{1}{2}\left(\frac{x-(-20)}{5}\right)^{2}} \\
\text { centro } & e^{-\frac{1}{2}\left(\frac{x-(0)}{5}\right)^{2}} \\
\text { menos }- \text { adelante } & e^{-\frac{1}{2}\left(\frac{x-(20)}{5}\right)^{2}} \\
\text { adelante } & e^{-\frac{1}{2}\left(\frac{x-(45)}{12}\right)^{2}}
\end{array}\right.
$$

Para la variable de salida que es el ángulo del torso con respecto a un punto de referencia, se definen tres funciones triangulares. Estas funciones son:

$$
y= \begin{cases}\text { menos }- \text { centro } & \frac{x-(-20)}{(-10)-(-20)}, \frac{20-x}{20-(-10)} \\ \text { centro } & \frac{x-(-20)}{(0)-(-20)}, \frac{20-x}{20-(0)} \\ \text { mas }- \text { centro } & \frac{x-(-20)}{(10)-(-20)}, \frac{20-x}{20-(10)}\end{cases}
$$

En base a las variables de entrada se define el grado de pertenencia a las funciones de la variable de salida. 


\subsection{Inferencia}

La inferencia se basa en el conocimiento de expertos mencionado en la sección Locomoción de Robots Bípedos, resultado del mismo análisis de la órbita del caminado. En el caminado, ambas piernas nunca tendrán la misma posición más que en la posición de inicio o reposo. En la Tabla 2 se muestran las posibles posiciones de las piernas de un caminado. En verde se muestran las posiciones posibles y en rojo las posiciones restringidas. En base a estas posiciones se definen las reglas difusas mediante proposiciones de Mamdani. Se busca compensar la posición del ángulo del torso para que no se aleje de cero. En color verde obscuro se muestran las posiciones de las piernas en las que la posición del ángulo del torso debe ser cero. En color verde claro se muestran las posiciones de las piernas en las que el ángulo debe compensarse para acercarlo a cero.

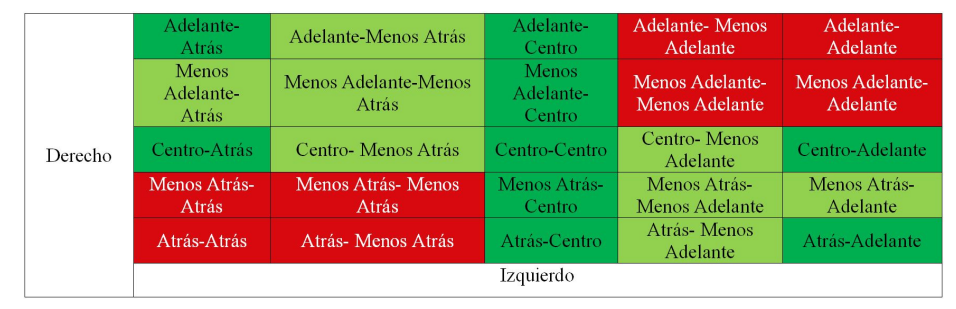

Fig. 2. Posición de las piernas en los pasos de un caminado.

En base a la Tabla 2 se establecieron 18 reglas difusas, de las cuales se muestran a continuación las nueve reglas pertenecientes a las sombreadas de color verde obscuro:

- IF (ángulo 1) is (centro) AND (ángulo 2) is (adelante) THEN (ángulo 3) is (centro)

- IF (ángulo 1) is (centro) AND (ángulo 2) is (centro) THEN (ángulo 3) is (centro)

- IF (ángulo 1) is (centro) AND (ángulo 2) is (atrás) THEN (ángulo 3) is (centro)

- IF (ángulo 1) is (adelante) AND (ángulo 2) is (centro) THEN (ángulo 3) is (centro)

- IF (ángulo 1) is (menos adelante) AND (ángulo 2) is (centro) THEN (ángulo 3 ) is (menos centro)

- IF (ángulo 1) is (menos atrás) AND (ángulo 2) is (centro) THEN (ángulo 3) is (mas centro)

- IF (ángulo 1) is (atrás) AND (ángulo 2) is (centro) THEN (ángulo 3) is (centro)

- IF (ángulo 1) is (adelante) AND (ángulo 2) is (atrás) THEN (ángulo 3) is (centro)

- IF (ángulo 1) is (atrás) AND (ángulo 2) is (adelante) THEN (ángulo 3) is (centro) 
Desarrollo de un sistema lógico difuso para el control de la locomoción bípeda de un robot ...

\subsection{Defuzzificación}

Para la defuzzificación se eligió el proceso de centroide, el cual es el promedio de todo el espacio de salida $y$, como se mencionó en la sección Control Óptimo Difuso, el método queda definido como en 11:

$$
y=\frac{\sum_{i=1}^{N} y_{i} \mu\left(y_{i}\right)}{\sum_{i=1}^{N} \mu\left(y_{i}\right)}, i=1,2, \ldots, n,
$$

donde $y$ será la salida única, $\mu\left(y_{i}\right)$ es el valor de pertenencia de $y_{i}$ en la función de membresía de la variable de salida.

\section{Pruebas y resultados}

Se hizo uso del toolbox de lógica difusa en MATLAB para el diseño del conrolador difuso del sistema definido en las secciones anteriores. Para demostrar el funcionamiento del control difuso se utilizó la herramienta Simulink de MATLAB y se realizó una simulación de en Webots con el robot humanoide NAO. En las siguientes secciones se ilustra el procedimiento realizado para el diseño de controlador difuso, la aplicación en Simulink y la simulación en Webots.

\subsection{Diseño del controlador difuso}

El diseño del controlador difuso se basa en lo definido en la sección de Desarrollo. En la Figura 3, se muestra el diagrama de bloques del controlador difuso para el sistema basado en las restricciones del modelo Walking Toy. Este controlador cuenta con dos entradas angulo1 y angulo2 para cada una de las piernas del bípedo. La salida del sistema es angulo3 el cual es el ángulo del torso con respecto a un punto de referencia que debe ser cercano a cero.

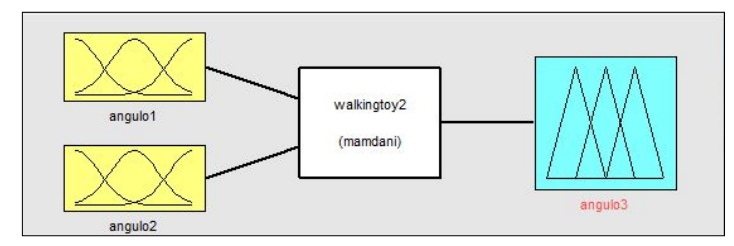

Fig. 3. Diagrama de bloques del controlador difuso para el sistema.

En la Figura 4 se muestran las funciones de membresía de las variables de entrada del sistema. Estas variables de entrada son los ángulos de las piernas que representan las posiciones de estas en el caminado. Cada variable cuenta con cinco funciones Gaussianas que fueron definidas con anterioridad. Estas funciones se definieron en un rango de $-45^{\circ}$ a $45^{\circ}$ la cual se consideran los grados 


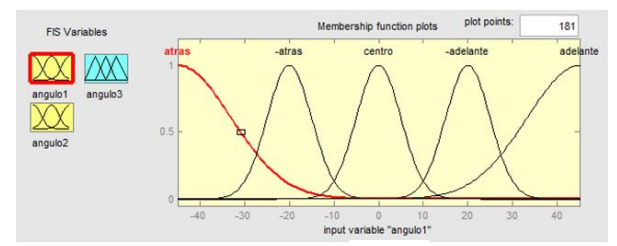

a)

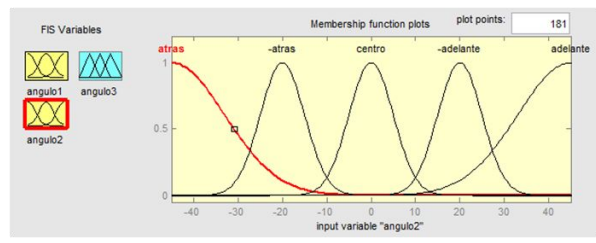

b)

Fig. 4. Muestra las funciones de membresía de las variables de entrada del sistema. (a) Variable ángulo 1, (b) Variable ángulo 2.

que pueden tomar las piernas. Las funciones son atrás, menos atrás, centro, menos adelante $y$ adelante.

En la Figura 5 se muestran las funciones de membresía de la variable de salida. Las funciones son triangulares y están definidas entre $-20^{\circ}$ a $20^{\circ}$. En este rango se encuentran los valores permitidos que puede tomar el ángulo del torso con respecto a un punto de referencia para mantenerlo cerca a cero. Estas funciones son menos centro, centro y mas centro. Los centro con modificadores lingüísticos se definen para poder compensar el sistema si este presenta mayor inclinación para cierto lado.

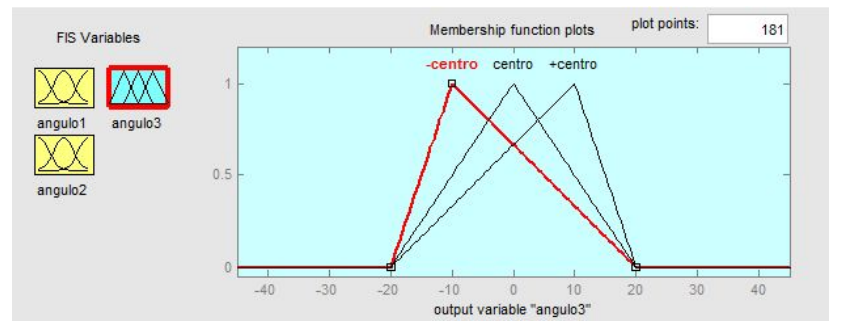

Fig. 5. Funciones de Membresía para la variable de salida del sistema.

Las reglas de inferencia del sistema se definen mediante proposiciones de Mamdani. Estas reglas buscan la aproximación al centro definido con el ángulo del torso. Además, buscan la compensación del torso al momento de efectuar pasos.

\subsection{Aplicación en Simulink}

El sistema se implemento en Simulink utilizando el toolbox de Lógica Difusa. Se definieron dos funciones sinusoidales con amplitud contraria para simular el ciclo del caminado para ambsa piernas. En la Figura 6 se muestra el diagrama de bloques de Simulink del sistema. En el diagrama de bloques del sistema, se 
Desarrollo de un sistema lógico difuso para el control de la locomoción bípeda de un robot ...

generan dos señales sinusoidales mediante el bloque Signal Generator, a cada uno se le agrega un offset de 0.5 para variar un poco la señal. Ambas señales se muestran en una gráfica con el bloque Scope. Estas señales entran al bloque Fuzzy Logic Controller el cual es el controlador difuso diseñado para el sistema con la aplicación de Lógica Difusa en MATLAB. La variable de salida se muestra en otra gráfica. En las Figuras 7 (a) y 7 (b) se muestran las señales de entrada sinusoidales y la señal de salida cercana a cero para el sistema del controlador difuso.

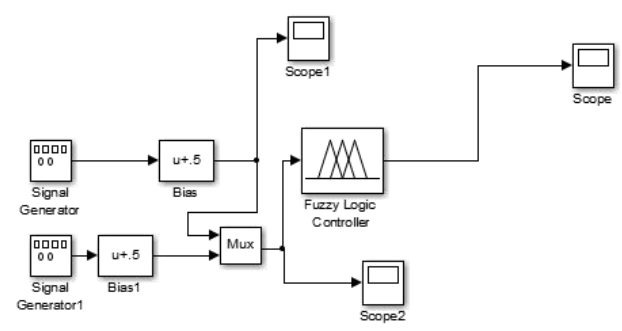

Fig. 6. Diagrama de bloques de Simulink del sistema.

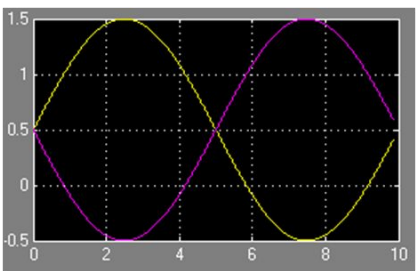

a)

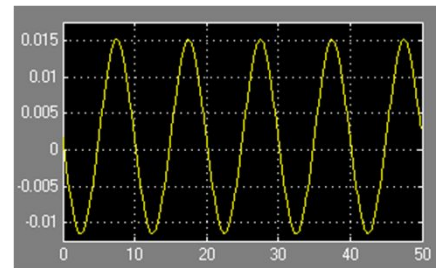

Fig. 7. (a) Variables de entrada representada en ciclos sinusoidales, (b) Señal de salida sinusoidal cercana a cero.

\subsection{Simulación}

La simulación del sistema lógico difuso para el control de la locomoción bípeda de un robot humanoide NAO se visualizó con el software Webots [1]. Este software es mundo virtual que permite lanzar una simulación de movimientos de NAO tomando en cuenta leyes físicas. Ofrece un lugar seguro para probar el funcionamiento de los comportamientos antes de reproducirlos en un robot 
real. Para realizar la simulación se recuperaron los ángulos de las trayectorias obtenidas con el controlador lógico difuso. Los ángulos de las piernas se utilizan para obtener la trayectoria lineal mediante una simple función trigonométrica:

$$
x=L \sin \varphi_{2},
$$

donde $x$ será la posición a colocar el pie del robot en el eje $x, L$ es la longitud de la pierna del robot, (se considera una longitud máxima de 200mm) y $\varphi_{2}$ es el ángulo de la pierna balanceada. La elevación de la pierna se considera de $50 \mathrm{~mm}$. Mientras que el ángulo del torso se compensa después de cada paso tomando el valor directamente del controlador difuso y pasandolo al robot. De manera cuantitativa se muestra en la Figura 8 la ejecución de un paso de 5 $\mathrm{cm}$ y una zancada de $10 \mathrm{~cm}$ por el método desarrollado en este trabajo y el control de Aldebaran. De esta comparativa lo que se puede resaltar es la similitud de estabilidad al avanzar el mismo tramo. Sin embargo, el control sobre la estabilidad del caminado utilizando el algoritmo de Aldebaran no es muy confiable en tramos más extensos, por este motivo se propone el uso del control lógico difuso. En la Figura 9 se muestra la secuencia de imágenes que representan un paso completo con ambas piernas realizado por el robot NAO. en la secuencia se observa que el torso se compensa al finalizar un paso con cada pierna.

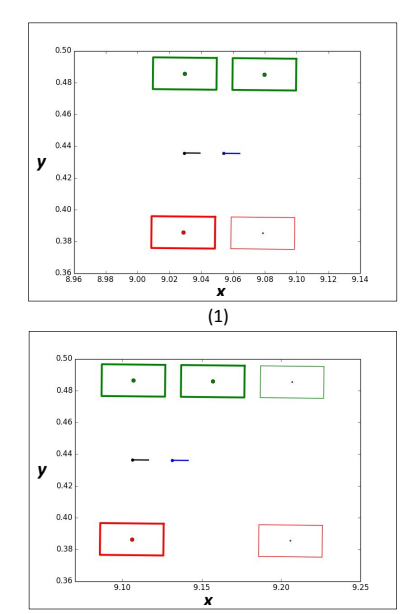

(3)
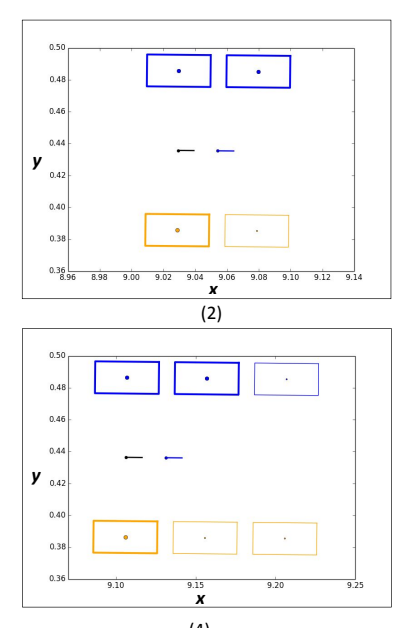

Fig. 8. Secuencia del caminado capturando la posición de los pies. (1) Un paso realizado mediante parámetros del controlador difuso. (2) Un paso realizado mediante el algoritmo de Aldebaran. (3) Una zancada realizada mediante parámetros del controlador difuso. (2) Una zancada realizada mediante el algoritmo de Aldebaran. 


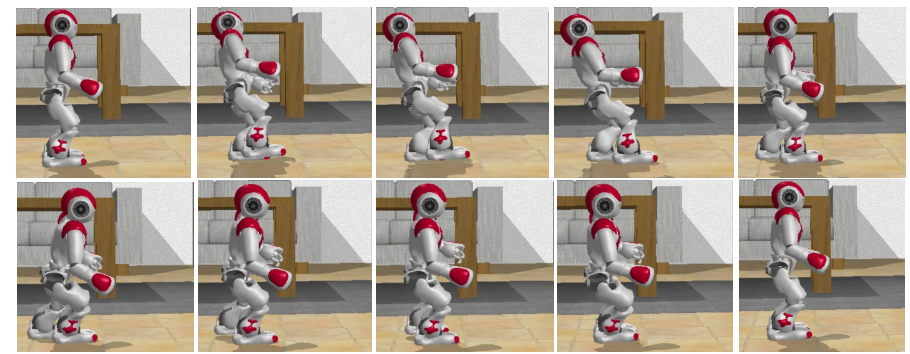

Fig. 9. Secuencia del caminado simulado en Webots por el robot NAO, se muestra un paso con el pie derecho y un paso con el pie izquierdo.

\section{Conclusiones}

Los sistemas de control convencionales son precisos y bien calculados para modelos matemáticos bien definidos. En sistemas donde no se conoce o no se puede modelar el sistema dinámico el control mediante lógica difusa es mejor opción. La razón por la que la lógica difusa será suficiente para poder controlar un sistema es porque el conocimiento que se utiliza es deducido por experiencia, observaciones y aplicaciones de los mismos. El razonamiento humano es difícil de modelar matemáticamente, este razonamiento se utiliza en la vida diaria y en cualquier evento cotidiano que describa una o unas acciones de un problema.

El sistema Walking Toy es un sistema dinámico que describe un modelo no lineal. Este sistema ha sido resuelto mediante control convencional con técnicas de control no lineal, sin embargo, no se conoce aplicación real. La resolución realizada en este trabajo fue una aproximación al control del caminado planteado por el método convencional. Los resultados obtenidos mostrados cumplieron con el objetivo de controlar el torso del bípedo para que se mantuviera cercano a la referencia cero. Como ventajas se tiene que el controlador difuso se implementa sin tanta complejidad. Fueron suficientes pocas reglas difusas para obtener un modelo robusto de estabilización del torso. El sistema es capaz de lidear con situaciones no previstas en la modelación. Entre las limitantes que tiene el sistema se puede mencionar la implementación. Esto debido a que solo se evalua con trayectorias rectas en un ambiente de simulación.

Como trabajos futuros se proponen la implementación del método convencional y lógica difusa con este sistema aplicado al robot humanoide NAO en una plataforma real. El objetivo de la implementación es la realización de una comparativa entre ambos controladores para verificar cuál de estos tiene mejores resultados en desempeño. Además de la implementación con trayectorias curvas y pendientes.

\section{Referencias}

1. ALDEBARAN, R.: Nao documentation (2015) 
2. Ankarali, A.: Fuzzy logic velocity control of a biped robot locomotion and simulation. Advanced Robotic Systems 9 (2012)

3. Azizi, V., Haghighat, A.T.: Fast and robust biped walking involving arm swing and control of inertia based on neural network whith harmony search optimizer. Methods and Models in Automation and Robotics (MMAR) (2011)

4. Czarmetzki, S., Jochmann, G., Kerner, S.: Nao devils dormound team description for robocup 2010. Tech. rep., Robotics Reserch Institute Section Information Technology TU Dormound University (2010)

5. Fernández-Iglesias, S.: Locomoción bípeda del robot humanoide nao. Tech. rep., Universidad Politécnica de Catalunya (November 2009)

6. Gorrostieta, E., Vargas-Soto, E.: Fuzzy algorithm of free locomotion for a six legged walking robot. Computación y Sistemas 11(3), 260-287 (2008)

7. Graf, C., Härtl, A., Rö, T., Laue, T.: A robust closed-loop gait for the standard platform league humanoid. In: Humanoid Soccer Robots. pp. 30-37. IEEE-RAS Intl. Conf On Humanoid Robots (December 2009)

8. Graf, C., Röfer, T.: A center of mass observing 3d-lipm gait for the robocup standard platform league humanoid. In: RoboCup 2011: Robot Soccer World Cup XV. pp. 102-113. Springer Berlin Heidelberg (2013)

9. Hengst, B.: Runswift walk2014 report robocup standard platform league. Tech. rep., School of Computer Science and Engineering University of New South Wale (2014)

10. Herdt, A.: Model predictive control of a humanoid robot. Ph.D. thesis, Insitut des sciences et technologies (2012)

11. Kitano, H., Asada, M., Kuniyoshi, Y., Noda, I., Osawa, E.: Robocup: the robot world cup initiative (1997)

12. Liu, Y., Wensing, P.M., Orin, D.E., Schmiedeler, J.P.: Fuzzy controlled hopping in a biped robot. Robotics and Automation (ICRA) (2011)

13. Massah, A., Sharifi, A., Salehinia, Y., Najafi, F.: An open loop walking on different slopes for nao humanoid robot. Proceedings Engineering pp. 296-304 (2012)

14. Rivas, F.M., Cañas, J.M., González, J.: Aprendizaje automático de modos de caminar para un robot humanoide. ResearchGate (2011)

15. Seven, U., Akbas, T., Fidan, K., Erbatur, K.: Bipedal robot walking control on inclined planes by fuzzy reference trajectory modification. Soft Comput (2012)

16. Sira-Ramírez, H., Agrawal, S.: The walking toy. In: Differentially Flat Systems, pp. 307-320. Marcel Dekker (April 2004)

17. Vargas-Soto, E., Gorrostieta, E., Sotomayor-Olmedo, A., Ramos-Arreguin, J., Tovar-Arriaga, S.: Design of fuzzy algorithms locomotion for six legged walking robot. International Journal of Physical Sciences 7(11), 1811-1819 (March 2012)

18. Yanjun, Z.: Fuzzy omnidirectional walking controller for the humanoid soccer robot. The 4th Workshop on humanoid Soccer Robots (2009)

19. Zaidi, A., Rokbani, N., Alimi, A.M.: A hierarchical fuzzy controller for a biped robot. Individual and Collective Behaviors in Robotics (ICBR) (2013) 